\title{
Usefulness of an acoustic edge artifact in assessment of the Ilizarov corticotomy interval
}

\author{
A. Zynamon, M.D., J.P. Crabbe, M.B., Ch. B., J.M. Rubin, M.D., Ph.D., and R.S. Adler, M.D., Ph.D. \\ Department of Radiology, University of Michigan Hospitals, Ann Arbor, Michigan, USA
}

\begin{abstract}
Thirty-three ultrasound examinations of the corticotomy interval of patients undergoing Ilizarov procedures were retrospectively evaluated for the presence or absence of an acoustic edge artifact. This artifact, consisting of a fine anechoic band, has been previously described in phantom models and is presumed to be due to phase cancellation effects. We demonstrated this artifact in 8 of 33 examinations. The artifact proved helpful in identifying the location of the corticotomy margin, even when this margin was obscured by the presence of developing periosteal new bone. Attention to technical factors is, however, important. We believe that this artifact may have a useful role in the routine monitoring of the Ilizarov patient.
\end{abstract}

Key words: Ultrasound - Bone - Ilizarov - Artifact Measurement

The first long-bone osteotomies for limb lengthening were performed by Codivilla in 1905 [2]. In 1952, Ilizarov introduced the technique that now bears his name - use of a mechanical device that allows controlled adjustment of the limb length and orientation [3]. This device is now used in a variety of applications, including the treatment of nonunion, infection, length discrepancy, and deformity. It offers many advantages over conventional methods of treatment of nonunion and limb lengthening. It is less invasive, has relatively infrequent complications, and allows immediate weight-bearing. The success of the treatment depends on a number of factors, including preservation of the extraosseous blood supply, stable fixation, time delay prior to distraction, and moderately stable distraction rates of $1 \mathrm{~mm}$ per day in frequent, small increments $[3,4,6]$.

Address reprint requests to: J.P. Crabbe, Department of Radiology, University of Michigan Hospitals, 1500 East Medical Center Drive, Ann Arbor, MI 48109, USA
Plain film radiographs play a key role in assessing the alignment and showing the development of new bone at the periosteal sleeve in the corticotomy gap. They are also used to monitor the progression of distraction at the corticotomy site.

Radiographic evaluation is, however, relatively insensitive in detecting small amounts of bone formation, and ultrasound has been reported to be the technique of choice in assessing the progression of new bone formation. This information permits adjustment in the rate of distraction at the corticotomy site in response to the rate of new bone formation [8]. Simultaneously, sonographic evaluation allows the assessment for complications of the Ilizarov technique, including the development of fluid collections, which may impede union or indicate infection.

Although there is some error tolerance in the measurement of the progress of limb distraction, particularly in children, the greater the precision of this measurement, the better will be the final outcome. Measurement of the corticotomy gap by plain radiography has the same inherent magnification problems as those wellknown in plain-film pelvimetry. Ultrasound measurements are corrected for magnification and therefore should lend themselves to the determination of the corticotomy interval. Furthermore, the corticotomy may be examined in its entirety, rather than in terms of a limited number of projections. Variations in the speed of sound secondary to soft-tissue heterogeneity can introduce errors in ultrasonographic measurement. Such inaccuracy would, however, occur in the direction of the beam, not perpendicular to it. Distances parallel to the transducer, such as the Ilizarov corticotomy interval, may be accurately determined.

Cortical bone typically appears as a strong specular reflector with an abrupt discontinuity at the corticotomy gap, but the development of periosteal new bone may obscure the corticotomy margin, making exact delineation of the corticotomy interval margins difficult.

In this paper, we describe an acoustic edge artifact that has the potential for precisely defining the cortico- 
tomy margin. This would improve the accuracy of ultrasonographic mensuration of the corticotomy interval and thereby increase the accuracy and usefulness of the routine sonographic monitoring of the Ilizarov technique.

\section{Materials and methods}

The edge artifact under study in this series appears as a narrow, sharply defined, hypoechoic shadow lying between the reverberation artifact seen behind the bone and the enhancement typically seen in the corticotomy interval. Its apex is directed towards the transducer and lies at the corticotomy margin, allowing the precise identification of this margin (Fig. 1).

Initially, to define and characterize the artifact, a contrast-detail phantom containing air-filled cylinders (described elsewhere [7]) was scanned using both a $3-\mathrm{MHz}$ and a $5-\mathrm{MHz}$ linear transducer with the transducer face parallel to the long axis of one of the cylinders. The effects of variable focal zone and transducer frequency were recorded, keeping other parameters (time gain compensation, overall gain, dynamic range, etc.) fixed.

Subsequently, a retrospective study was performed, consisting of 33 examinations of 14 patients (13 male, 1 female) obtained between November 1988 and October 1990. The age range was 6-37 years. All patients had the Ilizarov device applied to the lower extremity, 2 on the femur and 12 on the tibia/fibula. The elapsed interval following application of the device ranged from 5 to 19 months.

Indications for Ilizarov device application were correction of hypertrophic nonunion after fracture, 6 patients; limb lengthening for posttraumatic growth disturbances, 5 patients; correction of congenital deformities, 3 patients.

In all but 4 of the examinations, plain radiographs were obtained on the same day as the ultrasound scan and were reviewed for the presence or absence of new bone formation. In this retrospective study, undocumented variations in the radiographic technique precluded direct correlation of the radiographic and ultrasound measurements.

The clinical ultrasound studies were performed to determine the presence of fluid collections in the corticotomy interval, as part of the routine postoperative evaluation of the patient. An Acuson $5-\mathrm{MHz}$ linear array transducer was used, and studies were performed by a variety of operators. Patients were scanned with the transducer aligned parallel to the long axis of the bone. In this retrospective study, images were obtained with a variety of focal zone settings, some of which corresponded to the corticotomy margin.

Cases were reviewed for the presence or absence of such edge shadows at the corticotomy site. Since phantom studies indicate that this artifact is optimally visualized with the focal zone set at the level of the object margin, the location of the focal zone was recorded in each case. Also noted was the presence or absence of new bone formation in the corticotomy interval.

\section{Results}

On the phantom studies, the artifact was clearly and reproducibly visualized. The anechoic band was seen originating at the phantom margin when the focal zone was set at the level of the reflector. However, visualization of the artifact was found to be highly dependent on this focal zone setting (Fig. 2), with loss of the artifact when the focus was set below or above the specular reflector. Because of its greater penetration, the $3-\mathrm{MHz}$ transducer provided better visualization of the artifact than the $5-\mathrm{MHz}$ transducer. However, the artifact was reliably identified in the phantom using each transducer (Fig. 3).

No new bone was identified on the ultrasound study in 5 of the clinical cases, as these studies were performed as baseline examinations immediately following the creation of the corticotomy. All other examinations (28 cases) demonstrated new bone formation; in 9 of these examinations $(32 \%)$, new bone formation was not demonstrable on the plain radiographs.

On two occasions, difficulty was encountered in performing the ultrasound scan because of the overlying hardware; in another case, the corticotomy interval could not be measured as it was longer than the available transducer dimensions.

In measuring the corticotomy interval, the acoustic edge artifact was noted retrospectively. It was seen in 8 of the 33 examinations $(24 \%)$. In 5 of these, it was seen on both sides of the corticotomy, while in 3 , it was seen on only one side.

In 3 studies, the artifact was seen to a varying degree, despite the presence of developing new bone at the periosteal sleeve. Figure 4 demonstrates a clear example. In the remaining 5 studies in which the artifact was visualized, periosteal new bone had not as yet developed.

An attempt was made to determine the reason for the variability of the demonstration or failure to demonstrate the artifact. The focal distance setting was considered as a possible cause. In all cases in which the artifact was demonstrated, the focal zone was set within $2 \mathrm{~mm}$ proximal and $17 \mathrm{~mm}$ distal to the corticotomy, and in most of these the setting was at the corticotomy, or within $1 \mathrm{~cm}$ distal to it. When the artifact was not seen, the spread of the focal distance settings was much wider, ranging from $20 \mathrm{~mm}$ proximal to $40 \mathrm{~mm}$ distal to the corticotomy.

\section{Discussion}

Edge-shadow artifacts are commonly seen in B-mode ultrasound imaging, frequently when imaging the curved margins of fluid-filled structures. For linear transducers, a combination of reflection and refraction at the object/ surround interface is usually implicated as the underlying mechanism. Similar-appearing edge shadows have, however, been demonstrated in situations to which refraction/reflection arguments are not applicable.

In this study, we have demonstrated an edge shadow at the margins of the Ilizarov corticotomy site in 8 of 33 scans. Refraction cannot be implicated in the production of this artifact, as the edge effect involves an essentially perfect reflector through which no sound can pass.

Interference of the diffracted and transmitted waves at the margin of a reflector [5] could produce a discrete shadow, although the cylindrical nature of the diffracted wave would suggest that such a shadow would spread. This is in contrast to the thin discrete shadow observed in both phantom studies and in vivo. An alternative possibility is a model developed by Rubin et al. [7], and further elaborated by Adler et al. [1]. In this model, the 

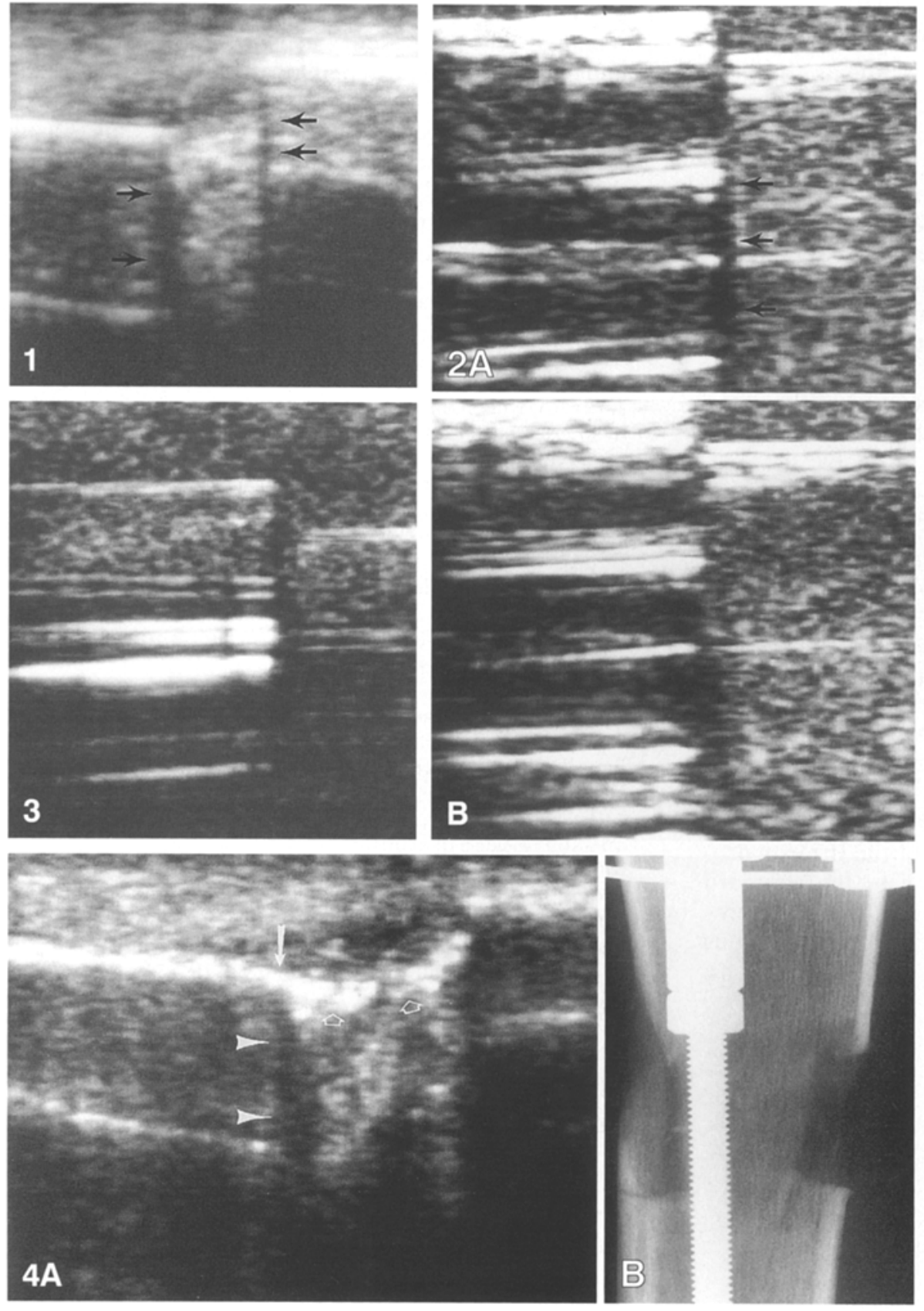

Fig. 1. Demonstration of the acoustic artifact (arrows) at each margin of the Ilizarov corticotomy

Fig. 2 A, B. The acoustic artifact at the margin of a perfect reflector in a phantom (3- $\mathrm{MHz}$ transducer), showing its dependence on focal zone setting. A The focal zone is set to the level of the reflector, and the artifact is clearly seen (arrows). B With the focal zone set $2 \mathrm{~cm}$ distal to the reflecting interface, the artifact is no longer demonstrated

Fig. 3. An identical phantom set-up to that of Fig. $2 \mathrm{~A}$, but with a transducer frequency of $5 \mathrm{MHz}$. The artifact is again demonstrated, despite reduced penetration

Fig. 4 A, B. The artifact identifies the corticotomy margin despite the presence of developing new bone in the periosteal sleeve. A Ultrasound section with the long axis of the $5-\mathrm{MHz}$ transducer parallel to the long axis of the tibia; long arrow, corticotomy margin; arrowheads, acoustic artifact; open arrows, developing new bone in the periosteal sleeve. B Corresponding radiograph shows developing periosteal new bone in the corticotomy interval concept of phase cancellation was introduced to explain the trailing hypoechoic band seen distal to the margin of a reflector. The large phase discontinuity at the finite transducer surface, situated along the line of tangency to the object margins (along the line of the clinically observed shadow) causes significant cancellation, thereby producing a thin linear shadow. This hypoechoic zone is seen at the junction of the reverberation artifact and the background speckle.

This seems a likely mechanism involved in the creation of the artifact we have observed in the ultrasonographic evaluation of the Ilizarov corticotomy: The bone edge essentially forms a sharp margin of a perfect reflector (bone) immediately adjacent to a transmitting medium (soft tissues), and the geometry of the interface may lead to the production of phase cancellation effects. In either case, the end result of such effects is the production of a shadow extending distally from the corticotomy margin. We have found that this shadow may be helpful in defining this margin.

Attention to technical factors is clearly important. A linear transducer is necessary to avoid divergence of the artifact. The artifact was best seen when the focal distance of the transducer was set to the corticotomy margin or within $1 \mathrm{~cm}$ distal to it; this dependence on focal zone setting was also clearly seen in the phantom images. In the phantom images, the artifact was demonstrated at two different transducer frequencies. In clini- 
cal practice, however, transducer frequency may also influence demonstration of the artifact: Although increased spatial resolution is achieved by the use of highfrequency transducers, the diminished penetration and loss of reverberation behind the bone may impair visualization of the artifact.

Ultrasonographic evaluation of the Ilizarov corticotomy is often performed to search for fluid collections in the region of the corticotomy defect. Moreover, ultrasound has been shown to be more sensitive than plain radiography in assessing the degree of new bone formation in the region. Also important in the radiologic evaluation of the Ilizarov procedure is the assessment of the amount of distraction that has occurred at the corticotomy site. An accurate measurement of this is important if the correct final limb length is to be attained. Plain film radiographs are routinely obtained, and estimates of the corticotomy interval can be made from these, particularly if magnification correction techniques are used. Ultrasound evaluation may lend itself well to mensuration of the corticotomy interval, but the presence of developing ossification in the sleeve of the periosteum may lead to difficulties in the precise localization of the corticotomy margins. We have found that the edge artifact described above can be seen despite the presence of developing new bone and can be used as a precise localizer of the corticotomy margin.

In conclusion, we have noted an edge artifact in our routine ultrasonographic monitoring of the Ilizarov procedure, which we feel may be of use in the standard ultrasonographic evaluation of the technique. A retrospective review of a series of ultrasound examinations of corticotomy intervals, performed for other purposes, has revealed that the finding is present in a significant percentage of cases, even when not being deliberately sought. We anticipate that if prospectively sought, the artifact would be visible in a greater percentage. Although we do not believe that this method of measuring the corticotomy interval will entirely replace plain film assessment, it is valuable in that it adds one more tool to our armamentarium in the radiologic assessment of the Ilizarov corticotomy.

\section{References}

1. Adler RS, Rubin JM, Fowlkes JB, Carson P (1991) Edge shadow by interference of diffracted and reflected waves in B-scan ultrasound images. J Ultrasound Med 10:S4

2. Codivilla A (1905) On the means of lengthening in the lower limbs, the muscles and tissues which are shortened through deformity. Am J Orthop Surg 2:353

3. Ilizarov $G$ (1952) A method of uniting bones in fractures and an apparatus to implement this method. USSR Authorship Certificate 98471

4. Ilizarov $G$ (1988) The principles of the Ilizarov method. Bull Hosp Jt Dis Orthop Inst 48:1

5. Morse PM, Ingard KU (1968) The scattering of sound. Diffraction from a knife-edge. In: Morse PM, Ingard KU (eds) Theoretical acoustics. Princeton University Press, Princeton, p 450

6. Paley D (1988) Current techniques of limb lengthening. J Pediatr Orthop 8:73

7.Rubin JM, Adler RS, Fowlkes JB, Carson PL (1991) Phase cancellation: a cause of acoustical shadowing at the edges of curved surfaces in B-mode ultrasound images. Ultrasound Med Biol $17: 85$

8. Young JWR, Kostrubiak IS, Resnik CS, Paley D (1990) Sonographic evaluation of bone production at the distraction site in Ilizarov limb-lengthening procedures. AJR 154:125 